\title{
EFEITO DA APLICAÇÃO DE CIANAMIDA HIDROGENADA E ÓLEO MINERAL NA QUEBRA DE DORMÊNCIA E PRODUCÃO DO PESSEGUEIRO-'FLAMECREST'’
}

\author{
GILMAR ARDUINO BETTIO MARODIN², IVAR ANTONIO SARTORI ${ }^{3}$, DENIS SALVATI GUERRA ${ }^{4}$
}

\begin{abstract}
RESUMO - Com o objetivo de aumentar a produção e qualidade de frutos do pessegueiro- 'Flamecrest', de alta exigência em frio, realizou-se um experimento no Município de Farroupilha-RS, no ano de 2000, com o uso de cianamida hidrogenada e óleo mineral. Estudou-se o efeito de duas diferentes épocas de aplicação dos tratamentos (15-7 e 2-8) sobre a brotação, floração, frutificação, produção e antecipação de colheita. Foram testados os efeitos dos seguintes tratamentos: cianamida hidrogenada (C.H.) 1,22; 2,45; 3,675 e 4,90 g de i.a.L-1 com óleo mineral (O.M.) $10 \mathrm{~g}$ de i.a.L $\mathrm{L}^{-1}$; O.M. $10 \mathrm{~g}$ de i.a. $\mathrm{L}^{-1}$ isolado e testemunha (sem pulverização). Os tratamentos não anteciparam a brotação das gemas floríferas, porém C.H. 2,45 e 4,90 g de i.a.L-1 com O.M. a 10 g de i.a.L-1 proporcionaram plantas com maior brotação das gemas vegetativas. Não houve efeito marcante dos tratamentos sobre a floração e a porcentagem de frutos vingados nas duas épocas. O número de frutos raleados por planta foi superior para os tratamentos com C.H. 2,45 e 4,90 g de i.a. $\mathrm{L}^{-1}$ com O.M. $10 \mathrm{~g}$ de i.a. $\mathrm{L}^{-1}$ quando aplicados na segunda época. Já, para o número e massa total dos frutos colhidos, destacaramse, na primeira época (15-7), os tratamentos com C.H. 1,22 e 3,675 g de i.a.L $\mathrm{L}^{-1}+$ O.M. $10 \mathrm{~g}$ de i.a.L $\mathrm{L}^{-1}$ e, na segunda época (2-8), os tratamentos com C.H. 2,45 e 4,90 g de i.a.L $\mathrm{L}^{-1}+$ O.M. $10 \mathrm{~g}$ de i.a.L $\mathrm{L}^{-1}$. Os tratamentos da segunda época resultaram em produções médias superiores à primeira. A massa média dos frutos não foi alterada de forma clara pelos tratamentos, assim como o teor de sólidos solúveis totais e a resistência de polpa.
\end{abstract}

Termos para indexação: Prunus persica, produtividade, dormência, antecipação de colheita.

\section{EFFECT OF THE APPLICATION OF HIDROGEN CYANAMID AND MINERAL OIL ON DORMANCY BREAKING AND PEACH PRODUCTION, CV. FLAMECREST}

\begin{abstract}
With the objective of increasing yields and fruit quality of cv. Flamecrest peaches, a cultivar with high chilling units demand, the present experiment was conducted in an orchard near to the Farroupilha city, in the year 2000. The hidrogen cyanamid and mineral oil were applied in two different times: middle of July and begining of August. The Dormancy breaking, flowering, fruit set, yield, and antecipation of harvest were evaluated from plants of the following treatments: 1,$22 ; 2,45 ; 3,675 \mathrm{or}^{4}, 90 \mathrm{~g}$ of i.a. $\mathrm{L}^{-1} \mathrm{hydrogen}$ cyanamid together with $10 \mathrm{~g}$ i.a L ${ }^{-1}$ of mineral oil or a single application of $10 \mathrm{~g} \mathrm{i.a} \mathrm{L}{ }^{-1}$ mineral oil. The Control plants were not sprayed with the dormancy breaking treatment. None of the treatments resulted in sprouting antecipation of flowering buds however, plants treated with 1,22 or $4,90 \mathrm{~g}$ i. $\mathrm{L} \mathrm{L}^{-1}$ hydrogen cyanamid mixed with $10 \mathrm{~g} \mathrm{i.a} \mathrm{L}{ }^{-1}$ mineral oil, had a higher number of sprouted vegetative buds. There was no significant effect of the period of application on flowering and fruit set. The number of fruits thinned out from each plant

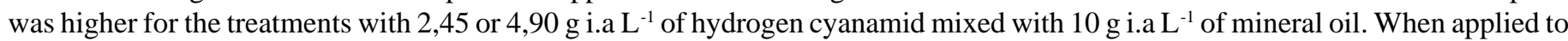
peaches trees in july, the treatments with 1,22 and 3,675 g i.a. $\mathrm{L}^{-1}$ of hydragen cyanamid and $10 \mathrm{~g} \mathrm{i.a.} \mathrm{L}^{-1}$ of mineral oil resulted in a higher number of fruits and total yield per plant. All the treatments, in the second apllication period, produced higher yields. The Average fruit weight, total soluble solids and pulp firmness were not influenced by the treatments.
\end{abstract}

Index terms: Prunus persica, dormancy, productivity, ripening anticipation.

\section{INTRODUÇÃO}

A produção mundial de pêssegos e nectarinas, no ano de 2000, foi da ordem de 13,8 milhões de toneladas, destacando-se a China, a Itália, os Estados Unidos e a Espanha. O Brasil aparece como o $13^{\circ}$ produtor mundial de pêssego, com 155 mil toneladas. O Rio Grande do Sul destaca-se como o maior produtor nacional, com aproximadamente 70 mil toneladas de pêssegos, sendo que a Metade Sul do Estado possui mais de 7 mil hectares, o que corresponde a $50 \%$ da produção gaúcha. No entanto, o Brasil ainda é um grande importador de frutas de caroço, tendo importado, entre os anos de 1997 e 1999, mais de 600 mil dólares em frutas frescas (Marodin \& Sartori, 2000).

A quebra de dormência das plantas caducifólias envolve fatores internos, como balanço dos promotores e inibidores de crescimento, ou externos, como temperatura, fotoperíodo e a radiação solar, entre outros. Dos fatores externos, o que mais se destaca é a temperatura, sendo que, quando as plantas são cultivadas em regiões com insuficiências de frio hibernal, apresentam sintomas de falta de adaptação como atraso e maior duração do período de floração e abertura de menor número de gemas floríferas e vegetativas, resultando em redução na produção,

1 (Trabalho 168/2001). Recebido: 16/10/2001. Aceito para publicação: 02/05/2002.

2 Eng ${ }^{\circ}$ Agr ${ }^{\circ}$, Dr., Prof. da Faculdade de Agronomia - UFRGS, CP 776, 91501-970, Porto Alegre - RS. E. mail: marodin@ vortex.ufrgs.br

3 Eng Agro $^{\circ}$, Msc., pela Faculdade de Agronomia da UFRGS, E-mail: ivar@ vortex.ufrgs.br

4 Eng ${ }^{\circ}$ Agr $^{\circ}$, Bolsista de Iniciação Científica, DHS, Faculdade de Agronomia da UFRGS.

Rev. Bras. Frutic., Jaboticabal - SP, v. 24, n. 2, p. 426-430, agosto 2002 
com frutos desuniformes e de baixa qualidade (Marodin et al., 1992).

Para completar sua formação, as gemas floríferas e vegetativas do pessegueiro devem atravessar um período de repouso, o qual está relacionado com o somatório de temperaturas abaixo de $7,2^{\circ} \mathrm{C}$. Constatou-se, porém, que temperaturas acima de $7,2^{\circ} \mathrm{C}$ também têm influência, principalmente em cultivares de menor exigência. A necessidade de frio do pessegueiro foi satisfeita com temperaturas médias de $9,7^{\circ} \mathrm{C}$. Cada cultivar tem suas exigências de horas de frio e horas de calor diferentes, o que explica como uma mesma cultivar tem florescimento em épocas distintas em locais diferentes (Diaz, 1987).

Em muitos anos, especialmente em regiões marginais para o cultivo de frutíferas temperadas, não ocorre a superação efetiva da dormência das gemas pela insuficiente acumulação de frio. Nestes casos, a utilização de produtos químicos é prática comum na viabilização destas espécies (Marodin et al., 1992).

Associações de óleo mineral e sais de dinitro, tais como DNOC, DNBP, calcionamida e nitrato de potássio, foram as mais utilizadas no Brasil na quebra da dormência das frutíferas temperadas (Petri, 1986). Porém, o uso dos sais de dinitro foi proibido, fazendo com que a melhor opção para a quebra de dormência passasse a ser a associação de cianamida hidrogenada $(\mathrm{CH}) \mathrm{com}$ óleo mineral ou, ainda, o nitrato de potássio (Araújo et al., 1991). Trabalhos de (Marodin et al., (1989) Petri et al. (1996) têm demonstrado que algumas cultivares de pêssego suportam maiores doses de $\mathrm{CH}$, mas o resultado é muito dependente da época de aplicação. Na cv. "Chiripá", concentrações acima de 0,25\% de $\mathrm{CH}$, próximo da floração, causou intensa queda de gemas floríferas, sem prejuízo da produção total (Marodin et al., 1989).

Uma série de fatores, como a concentração dos produtos, épocas de aplicação, condições meteorológicas, estádio das gemas, volume de solução aplicado por planta, somatório das horas de frio acumuladas pela planta, devem ser considerados na quebra de dormência, sendo necessários trabalhos para identificar tais fatores para cada local e cultivar (Marodin et al., 1991).

Na cv. Rubidoux, a produção e número de frutos por planta, em Videira-SC, foi muito dependente das concentrações de $\mathrm{CH}$, ocorrendo queda de produção em concentrações superiores à normal. A antecipação de colheita de cerca de 15 dias foi significativa com baixas concentrações de CH (Petri et al., 1996).

No México, tratamentos de $\mathrm{CH}+$ extrato de alho ou + OM aplicados isolados ou combinados, em ameixeiras-japonesas "Shiro", anteciparam a brotação e a colheita em torno de 15 dias. A mais elevada percentagem de brotação vegetativa foi alcançada com as concentrações médias de $\mathrm{CH}$ e OM. Os tratamentos com $\mathrm{CH}$ resultaram em maior intensidade de floração, menor queda de gemas florais e maior produção por planta (Marodin et al., 1997).

Em pessegueiros-“Chiripá”, C.H. 0,75\% com O.M. 1,0\% propiciou um aumento no número de frutos por plantas e no peso dos frutos. O.M. 2,0\% ficou numa faixa intermediária entre a maior e a menor produção, não sendo, porém, significativa a diferença em relação aos outros tratamentos e à testemunha (Nunes et al., 2001).

A produção de frutas, em especial de pêssegos e nectarinas na região da Serra do Nordeste do Rio Grande do Sul, onde foi realizado o presente estudo, é de extrema importância para a pequena propriedade rural. Produtores têm buscado alternativas de cultivares com melhor adaptação, qualidade dos frutos e possibilidade de armazenamento. Estudos preliminares mostraram que as cvs. Flavor Crest, Elegant Lady, Tropic Snow e Flamecrest têm potencial de cultivo (Perazzolo, 1999), mas têmse observado problemas de uniformidade de brotação e produções insuficientes. O objetivo deste trabalho foi estudar os efeitos de aplicações de diferentes doses de cianamida hidrogenada e óleo mineral sobre a brotação vegetativa, frutificação e qualidade dos frutos do pessegueiro-'Flamecrest', uma das cultivares mais promissoras devido à época de colheita.

\section{MATERIAL E MÉTODOS}

O experimento foi realizado no Município de FarroupilhaRS, no ano de 2000, em um solo Cambissol Húmico álico textura argilosa (Cambissolo Húmico Alumínico Típico - EMBRAPA, 1999) com altitude de 702 metros do nível do mar, latitude $29^{\circ} 14^{\prime}$ $30^{\prime \prime}$ e longitude $51^{\circ} 26^{\prime} 20^{\prime \prime}$ com temperaturas máximas de 35,2 e mínimas de $-6,6^{\circ} \mathrm{C}$. O clima da região é do tipo $\mathrm{Cfb}$, subtropical chuvoso com verão ameno (Köppen, 1948). A cultivar estudada foi o pessegueiro- 'Flamecrest', de alta exigência de frio, enxertada sobre o porta-enxerto capdeboscq, em um pomar comercial com 4 anos de idade, com espaçamento entre filas de $5 \mathrm{~m}$ e $2,5 \mathrm{~m}$ entre plantas.

O delineamento experimental adotado foi de blocos casualizados, com uma planta por parcela e 4 repetições. Planta bordadura foi utilizada somente para separar os blocos. A aplicação dos tratamentos foi realizada com pulverizador costal manual, com pressão de $70 \mathrm{Lb} /$ pol, sendo na primeira aplicação com temperaturas próximas de zero grau e na segunda em torno de $15^{\circ} \mathrm{C}$. O volume médio de calda aplicado por planta foi de 1,2 litro. Fez-se uso de uma lona plástica para evitar a deriva da aplicação às plantas ao lado.

Foram testados os efeitos dos seguintes tratamentos: Cianamida hidrogenada (C.H.) 1,22; 2,45; 3,675 e 4,90 g de i.a.L ${ }^{-1}$ com óleo mineral (O.M.) $10 \mathrm{~g}$ de i.a.L $\mathrm{L}^{-1}$; O.M. $10 \mathrm{~g}$ de i.a. $\mathrm{L}^{-1}$ isolado e testemunha (sem pulverização), em duas épocas de aplicação, 15-07 (plena dormência) e 02-08 (próximo do inchamento das gemas).

Foram obtidos dados de brotação de gemas vegetativas e florais, através da marcação e contagem de gemas em quatro ramos, nos quadrantes de cada planta, e calculados em porcentagem. Acompanhou-se a fenologia das plantas e avaliou-se a fitotoxicidade.

As variáveis avaliadas foram o número de frutos raleados e o número, a massa, o teor de sólidos solúveis totais (SST) e a firmeza da polpa dos frutos colhidos. Foram retiradas amostras de 10 frutos por tratamento para determinar o teor de sólidos solúveis totais (SST) com o uso de refratômetro de mesa e firmeza de polpa em (Lb/pol) avaliada por meio de um penetrômetro FT-11, utilizando uma ponta de $5 \mathrm{~mm}$ de diâmetro.

Os dados foram processados para análise de variância do Sistema Sanest e, quando da significância, realizou-se o Teste de Duncan, com nível de significância de 5\%, para separar as médias. 


\section{RESULTADOS E DISCUSSÃO}

Os dados de acumulação de frio de maio a setembro, coletados a poucos quilômetros do pomar estudado, indicaram um somatório de 758 horas abaixo de $7,2 \mathrm{C}^{\circ}$, teoricamente suficiente para a adequada quebra de dormência desta cultivar, o que não ocorre na prática, indicando que este não é o melhor meio para avaliar a adaptação da maioria das frutíferas temperadas.

Os tratamentos não anteciparam a brotação das gemas floríferas, porém plantas tratadas com C.H. 1,22 g i.a. $\mathrm{L}^{-1} \mathrm{e} 3,675 \mathrm{~g}$ i.a. $\mathrm{L}^{-1}$, na primeira época, e $\mathrm{CH} 2,45$ e 4,90 g i.a. $\mathrm{L}^{-1}$, na segunda época, acrescidas de O.M. $10 \mathrm{~g}$ de i.a. $\mathrm{L}^{-1}$, tiveram maior brotação das gemas vegetativas em relação aos demais tratamentos (Tabela 1). Estes resultados estão de acordo com Nunes et al. (2000), que também não encontraram antecipação das gemas vegetativas no pessegueiro- "Chiripá" utilizando diversas concentrações de CH. Marodin et al. (1997), na ameixeira híbrida 'Shiro', verificaram uma antecipação na brotação das gemas vegetativas em 15 dias, com CH a 0,245\% com ou sem OM. Parece que a espécie e/ ou cultivar e as condições meteorológicas pós-aplicação têm grande influência na brotação das fruteiras de caroço.

Não houve efeito dos tratamentos sobre a floração e a porcentagem de frutos vingados, discordando dos dados obtidos por Marodin et al. (1997), onde os tratamentos que incluíam CH incrementaram a intensidade de floração da ameixeira- 'Shiro'. Fato relevante foi a ausência de efeito fitotóxico às flores quando se utilizaram concentrações superiores a 3,675 g de i.a. $L^{-1} \mathrm{de}$ $\mathrm{CH}$, pois já se havia constatado que menores concentrações, de cerca de 2,45 g i.a. $\mathrm{L}^{-1}$ de $\mathrm{CH}$, provocaram queda de gemas e redução da frutificação no pessegueiro- ' Chiripá' (Marodin et al., 1989).

O número de frutos raleados por planta foi maior nos tratamentos com C.H. 3,675 g de i.a.L $\mathrm{L}^{-1}+$ O.M. $10 \mathrm{~g}$ de i.a. $\mathrm{L}^{-1}$ na primeira época e $\mathrm{CH} 2,45$ e $4,90 \mathrm{~g}$ de i.a. $\mathrm{L}^{-1}+$ O.M. $10 \mathrm{~g}$ de i.a. $\mathrm{L}^{-1}$ na segunda época estudada (Tabela 2), concordando com Marodin et al. (1997) e Nunes et al. (2000) que encontraram menor queda

TABELA 1 - Porcentagem de gemas vegetativas e floríferas vingadas do pessegueiro-“Flamecrest” para a época 1 (15-7) e época 2 (28) em Farroupilha-RS, 2000.

\begin{tabular}{|c|c|c|c|c|}
\hline \multirow[t]{2}{*}{ Tratamentos } & \multicolumn{2}{|c|}{ Brotação de gemas vegetativas } & \multicolumn{2}{|c|}{ Floração } \\
\hline & Época 1 & Época 2 & Época 1 & Época 2 \\
\hline $\mathrm{CH} 1,22$ g i.a. $\mathrm{L}^{-1}+$ O.M. $10 \mathrm{~g}$ i.a. $\mathrm{L}^{-1}$ & A $25,50 \mathrm{a}$ & $\mathrm{C} 22,75 \mathrm{a}$ & 29,25 & 31,50 \\
\hline $\mathrm{CH} 2,45$ g i.a. $\mathrm{L}^{-1}+$ O.M. $10 \mathrm{~g}$ i.a. $\mathrm{L}^{-1}$ & $\mathrm{BC} 18,25 \mathrm{~b}$ & A $38,00 \mathrm{a}$ & 25,75 & 37,00 \\
\hline $\mathrm{CH}$ 3,675 g i.a. $\mathrm{L}^{-1}+$ O.M. $10 \mathrm{~g}$ i.a. $\mathrm{L}^{-1}$ & $\mathrm{AB} 21,75 \mathrm{a}$ & B $30,75 \mathrm{a}$ & 33,25 & 30,75 \\
\hline $\mathrm{CH} 4,90$ g i.a. $\mathrm{L}^{-1}+$ O.M. 10 g i.a. $\mathrm{L}^{-1}$ & não realizado & A $40,00 \mathrm{a}$ & não realizado & 24,75 \\
\hline O.M. 10 g i.a.L $\mathrm{L}^{-1}$ & $\mathrm{C} 14,25 \mathrm{a}$ & D $8,75 \mathrm{a}$ & 27,75 & 21,50 \\
\hline Testemunha & D $10,00 \mathrm{a}$ & D $6,25 \mathrm{a}$ & 25,75 & 30,00 \\
\hline Média Geral & 17,95 & 24,42 & 28,35 & 29,25 \\
\hline $\mathrm{F}$ & $* * 27,21$ & $* * 9,44$ & $0,93 \mathrm{~ns}$ & $1,08 \mathrm{~ns}$ \\
\hline $\mathrm{CV} \%$ & 17,62 & 25,15 & 19,27 & 36,69 \\
\hline
\end{tabular}

Médias antecedidas por letras maiúsculas distintas, na coluna, e letras minúsculas, na linha, diferem entre si, ao nível de significância de 5 \%, pelo teste de Duncan.

Obs: Tratamento não realizado.

* Significativo a $5 \%$ de probabilidade.

** Significativo a $1 \%$ de probabilidade.

ns: não significativo.

TABELA 2 - Número de frutos vingados/ramo e raleados/planta do pessegueiro-"Flamecrest" para a época 1 (15-7) e época 2 (2-8) em Farroupilha-RS, 2000.

\begin{tabular}{|c|c|c|c|c|}
\hline \multirow[t]{2}{*}{ Tratamentos } & \multicolumn{2}{|c|}{ Frutos vingados/ramo } & \multicolumn{2}{|c|}{ Frutos raleados/planta } \\
\hline & Época 1 & Época 2 & Época 1 & Época 2 \\
\hline & \multicolumn{2}{|c|}{ —___ Número-___ } & $\ldots$ & - _- \\
\hline CH 1,22 g i.a. $L^{-1}+$ O.M. 10 g i.a. $L^{-1}$ & 09,65 & 8,71 & B $28,25 \mathrm{~b}$ & B $50,25 \mathrm{a}$ \\
\hline $\mathrm{CH} 2,45$ g i.a. $\mathrm{L}^{-1}+$ O.M. 10 g i.a. $\mathrm{L}^{-1}$ & 06,73 & 7,87 & $\mathrm{C} 21,25 \mathrm{~b}$ & A 84,25 a \\
\hline $\mathrm{CH} 3,675$ g i.a. $L^{-1}+$ O.M. 10 g i.a. $L^{-1}$ & 10,08 & 7,38 & A 40,75 a & B $44,00 \mathrm{a}$ \\
\hline CH 4,90 g i.a. $L^{-1}+$ O.M. 10 g i.a. $L^{-1}$ & não realizado & 7,08 & não realizado & A $98,00 \mathrm{a}$ \\
\hline O.M. 10 g i.a. $L^{-1}$ & 06,68 & 5,85 & C $16,50 \mathrm{a}$ & D $13,25 \mathrm{a}$ \\
\hline Testemunha & 04,96 & 5,45 & $\mathrm{C} 15,00 \mathrm{~b}$ & C $25,00 \mathrm{a}$ \\
\hline Média Geral & 7,62 & 7,06 & 24,35 & 52,45 \\
\hline $\mathrm{F}$ & $2,99 \mathrm{~ns}$ & $0,01 \mathrm{~ns}$ & $* * 65,44$ & $* * 35,74$ \\
\hline $\mathrm{CV} \%$ & 31,74 & 32,25 & 13,37 & 19,10 \\
\hline
\end{tabular}

Médias antecedidas por letras maiúsculas distintas, na coluna, e letras minúsculas, na linha, diferem entre si, ao nível de significância de 5 \%, pelo teste de Duncan.

* Significativo a $5 \%$ de probabilidade.

** Significativo a $1 \%$ de probabilidade.

ns: não significativo. 
TABELA 3 - Número e massa (kg/planta) de frutos, peso médio dos frutos (g) do pessegueiro-“Flamecrest" para a época 1 (15-7) e época 2 (2-8) em Farroupilha-RS, 2000.

\begin{tabular}{|c|c|c|c|c|c|c|}
\hline \multirow[t]{2}{*}{ Tratamentos } & \multicolumn{2}{|c|}{ Frutos } & \multicolumn{2}{|c|}{ Massa de frutos } & \multicolumn{2}{|c|}{ Massa média dos frutos } \\
\hline & Época 1 & Época 2 & Época 1 & Época 2 & Época 1 & Época 2 \\
\hline & \multicolumn{2}{|c|}{ —-Número-_-_- } & \multicolumn{2}{|c|}{$\longrightarrow(\mathrm{kg} / \mathrm{pl})$} & \multicolumn{2}{|c|}{$\longrightarrow(\mathrm{g} /) \longrightarrow$} \\
\hline CH 1,22 g i.a. $L^{-1}+$ O.M. 10 g i.a. $L^{-1}$ & A $83,75 b$ & B 126,00 a & A $10,10 \mathrm{~b}$ & B 14,73 a & A 129,84 a & B $116,76 b$ \\
\hline $\mathrm{CH} 2,45$ g i.a. $\mathrm{L}^{-1}+$ O.M. 10 g i.a.L $\mathrm{L}^{-1}$ & B $57,75 \mathrm{~b}$ & A 153,50 a & B $06,68 \mathrm{~b}$ & A 17,97 a & C 119,10 a & B $116,98 \mathrm{~b}$ \\
\hline CH 3,675 g i.a. $. L^{-1}+$ O.M. 10 g i.a. $L^{-1}$ & A $88,00 \mathrm{~b}$ & CD 101,50 a & A 10,78 a & $\mathrm{C} 12,13 \mathrm{a}$ & B 126,88 a & A 120,25 a \\
\hline $\mathrm{CH}$ 4,90 g i.a. $\mathrm{L}^{-1}+$ O.M. 10 g i.a. $\mathrm{L}^{-1}$ & não realizado & A 158,50 a & não realizado & A 18,35 a & não realizado & A 124,19 a \\
\hline O.M. 10 g i.a. $\mathrm{L}^{-1}$ & B $57,00 \mathrm{~b}$ & E 73,25 a & B 06,68 a & D $8,54 \mathrm{a}$ & B 126,58 a & B $116,81 \mathrm{~b}$ \\
\hline Testemunha & BC $50,75 \mathrm{~b}$ & C 95,50 a & B $06,10 \mathrm{~b}$ & C $12,04 \mathrm{a}$ & BC 124,32 a & C $112,01 \mathrm{~b}$ \\
\hline Média Geral & 67,45 & 118,04 & 8,07 & 13,96 & 125,34 & 117,83 \\
\hline $\mathrm{F}$ & $* * 29,32$ & $* * 46,82$ & $* * 15,84$ & 9,65 & $* 2,81$ & $* 2,87$ \\
\hline $\mathrm{CV} \%$ & 7,47 & 7,19 & 10,43 & 15,74 & 3,58 & 4,80 \\
\hline
\end{tabular}

Médias antecedidas por letras maiúsculas distintas, na coluna, e letras minúsculas, na linha, diferem entre si, ao nível de significância de $5 \%$, pelo teste de Duncan.

* Significativo a $5 \%$ de probabilidade.

** Significativo a $1 \%$ de probabilidade.

ns: não significativo.

TABELA 4 - Teor de sólidos solúveis totais e firmeza de polpa do pessegueiro-“Flamecrest” para a época 1 (15-7) e época 2 (2-8) em Farroupilha-RS, 2000.

\begin{tabular}{|c|c|c|c|c|}
\hline \multirow[t]{2}{*}{ Tratamentos } & \multicolumn{2}{|c|}{ Teor de SST } & \multicolumn{2}{|c|}{ Firmeza de polpa } \\
\hline & Época 1 & Época 2 & Época 1 & Época 2 \\
\hline & \multicolumn{2}{|c|}{${ }^{-}{ }^{\circ} \mathrm{Brix} \longrightarrow$} & \multicolumn{2}{|c|}{$\longrightarrow$ Lb/pol- } \\
\hline $\mathrm{CH} 1,22$ g i.a. $\mathrm{L}^{-1}+$ O.M. 10 g i.a. $\mathrm{L}^{-1}$ & 7,40 & 7,80 & $\mathrm{AB} 4,0 \mathrm{a}$ & $\mathrm{C} 4,3 \mathrm{a}$ \\
\hline CH 2,45 g i.a. $\mathrm{L}^{-1}+$ O.M. 10 g i.a. $\mathrm{L}^{-1}$ & 8,10 & 8,19 & A 4,2 a & D 3,3 a \\
\hline CH 3,675 g i.a.L $\mathrm{L}^{-1}+$ O.M. 10 g i.a. $\mathrm{L}^{-1}$ & 7,76 & 7,73 & $\mathrm{BC} 3,5 \mathrm{~b}$ & A 6,3 a \\
\hline CH 4,90 g i.a.L $\mathrm{L}^{-1}$ + O.M. 10 g i.a. $\mathrm{L}^{-1}$ & não realizado & 8,00 & não realizado & D $3,2 \mathrm{a}$ \\
\hline O.M. 10 g i.a. $L^{-1}$ & 7,53 & 7,60 & BC 3,3 a & $\mathrm{C} 4,2 \mathrm{a}$ \\
\hline Testemunha & 8,53 & 7,26 & $\mathrm{C} 3,0 \mathrm{~b}$ & B 5,3 a \\
\hline Média Geral & $\mathbf{7 , 8 6}$ & 7,76 & 3,60 & 4,43 \\
\hline $\mathrm{F}$ & $1,45 \mathrm{~ns}$ & $2,88 \mathrm{~ns}$ & $* 2,01$ & $* 5,98$ \\
\hline $\mathrm{CV} \%$ & 6,11 & 6,93 & 13,57 & 17,01 \\
\hline
\end{tabular}

Médias antecedidas por letras maiúsculas distintas, na coluna, e letras minúsculas, na linha, diferem entre si, ao nível de significância de $5 \%$, pelo teste de Duncan.

* Significativo a $5 \%$ de probabilidade.

** Significativo a $1 \%$ de probabilidade.

ns: não significativo.

de floração e, conseqüentemente, aumento da frutificação com aplicações da cianamida em concentrações em torno de C.H. 2,45 g i.a. $L^{-1}$, associadas ao óleo mineral.

A produção de frutos por planta está colocada na Tabela 3. Constata-se que, para o número e peso total dos frutos, os tratamentos com C.H. 1,22 g i.a. $L^{-1}$ e 3,675 g i.a. $L^{-1}$, aplicados na primeira época (15-7), e C.H. 3,675 e 4,90 g i.a..$^{-1}$ na segunda época (2-08), todos com O.M. $10 \mathrm{~g}$ de i.a. $\mathrm{L}^{-1}$, proporcionaram valores significativamente maiores aos demais tratamentos, concordando com Marodin et al. (1997; Nunes et al. (2000) e Petri et al. (1996), que encontraram maior produção de frutos por planta em concentrações similares. Os tratamentos aplicados na segunda época, na média, foram maiores, inclusive na testemunha, provavelmente, devido ao efeito de ventos, já que as plantas da primeira época estavam na primeira fila do pomar e sujeitas a maior intensidade de ventos. Isto ocorreu porque o trabalho foi realizado em pomar comercial, não sendo possível a escolha ale- atória. Quanto ao peso médio dos frutos, na segunda época, houve destaque dos tratamentos com C.H. 3,675 e $4,90 \mathrm{~g}$ de i.a.L ${ }^{1}$ + O.M. $10 \mathrm{~g}$ de i.a. $\mathrm{L}^{-1}$, não se constatando praticamente nenhum efeito no tamanho dos frutos na primeira época (Tabela 3).

O teor de sólidos solúveis totais não foi alterado por todos os tratamentos nas duas épocas, concordando com a maioria dos trabalhos onde a quebra de dormência não afeta as características das frutas. No entanto, a resistência de polpa foi superior nos tratamentos com CH 1,22 g i.a. $\mathrm{L}^{-1}$ e 2,45 g i.a. $\mathrm{L}^{-1}$, na primeira época, e CH 3,675 g i.a. $\mathrm{L}^{-1}$, na segunda, provavelmente, pelo tamanho da amostra de 10 frutos ser insuficiente para esta avaliação (Tabela 4).

Os tratamentos com O.M. $10 \mathrm{~g}$ de i.a. $\mathrm{L}^{-1}$ não resultaram em aumentos significativos para todas as variáveis estudadas em relação à testemunha. Também não foi constatada antecipação de colheita para todos os tratamentos testados, ao contrário do que havia sido encontrado por Petri et al. (1996). 


\section{CONCLUSÕES}

1. Os tratamentos não afetaram a floração, mas a brotação das gemas vegetativas foi incrementada com C.H. 2,45 e 4,90 g de i.a. $\mathrm{L}^{-1}$ associados ao O.M. a $10 \mathrm{~g}$ de i.a. $\mathrm{L}^{-1}$, principalmente quando aplicados na segunda época, cerca de 40 dias antes da plena florada.

2. Para a primeira época (15-7), os tratamentos com C.H. 1,22 e 3,675 g i.a.L $\mathrm{L}^{-1}$ com O.M. $10 \mathrm{~g}$ de i.a.L-1 proporcionaram maior produção e, para a segunda época (2-8), C.H. 2,45 e 4,90 g de i.a. $\mathrm{L}^{-1}$ com O.M. $10 \mathrm{~g}$ de i.a. $\mathrm{L}^{-1}$.

3. O teor se sólidos solúveis totais não foi alterado pelos tratamentos.

4. Os tratamentos com O.M. $10 \mathrm{~g}$ de i.a. $\mathrm{L}^{-1}$ não proporcionaram aumentos significativos nas variáveis estudadas.

5. Não houve efeitos de fitotoxicidade nas plantas tratadas, como queda de gemas florais, mesmo nas concentrações de $\mathrm{CH} 4,90 \mathrm{~g}$ de i.a. $\mathrm{L}^{-1}+$ O.M. $10 \mathrm{~g}$ de i.a. $\mathrm{L}^{-1}$.

\section{REFERÊNCIAS BIBLIOGRÁFICAS}

ALVARADO R.H. et al. El Thidiazurón, La Brotación Floral y las dimenciones Del ovário en ciruelo japonés (Prunus salicina L.) 'Shiro'. Agrociencia, v.34, s/n., p. 321-327, 2000.

ARAÚJO, M. M.; FORTES, G. R. de L.; SANTOS FILHO, B. Thidiazuron: uma alternativa para superar a dormência de gemas de macieira (Malus domestica, Borkh). Revista Brasileira de Fruticultura, Cruz das Almas, v.223, n.3, p. 249-253, 1991.

DIAZ, M. D. H. Requerimento de frio em frutales caducifolios. Instituto Nacional de Investigaciones. Forestales y Agropecuarios. México: SARH, 1987. 55 p.(Tema, 2).

EMBRAPA. Centro Nacional de Pesquisa de Solos. Sistema brasileiro de classificação de solos. Brasília: EMBRAPA. Rio de Janeiro. 412p.

MARODIN, G. A. B.; LUCHESE, O. A.; MANFROI, V.; GERHARDT, I. R.; AMARO, S.S. A Cianamida hidrogenada e Óleo Mineral na quebra da dormência e produção do pessegueiro cv. "Chiripá". In: CONGRESSO BRASILEIRO DE FRUTICULTURA, 10, 1989. Fortaleza, CE, Resumos... Sociedade Brasileira de Fruticultura, 1989.56 p.
MARODIN, G. A. B.; LUCHESE, O. A.; MANFROI, V. Efeito da aplicação de Cianamida Hidrogenada e do Óleo Mineral na quebra de dormência e antecipação de colheita do pessegueiro "Premier". Revista Brasileira de Fruticultura, Cruz das Almas, v. 13, n. 3, p. 165 - 171, 1991.

MARODIN, G. A. B.; FRANCISCONI, A. H. D.; GALLOIS, E. S.P. Efeito de produtos químicos na quebra de dormência e produção de Pereira (Pyrus communis, L.) cv Packham's Triumph. Revista Brasileira de Fruticultura, Cruz das Almas, v.14, n.1, p.155 - 160, 1992.

MARODIN, G. A. B.; ROMÁN, A.E.B. Cianamida Hidrogenada e Óleo Mineral e o extrato de alho na quebra de dormência e produção da ameixeira cv. "Shiro" em Texcoco - México. Pesquisa Agropecuária Gaúcha, v.3, n.2, p. 177-181, 1997.

MARODIN, G.A.B.; SARTORI, A.S.; Situação das frutas de caroço no Brasil e no mundo. In. SIMPÓSIO INTERNACIONAL DEFRUTAS DECAROÇO: PÊSSEGOS, NECTARINAS EAMEIXAS, 1., 2000, Porto Alegre. Anais... Porto Alegre: UFRGS-DHS, 2000. p. 7-16.

NUNES, J.L. da S.; MARODIN, G. A. B.; SARTORI, I.A. Cianamida Hidrogenada, Thidiazuron e Óleo Mineral na quebra da dormência e na produção do pessegueiro cv. "Chiripá". Revista Brasileira de Fruticultura, Jaboticabal, v. 23, n.. 3, p. 493-496, 2001.

KOPPEN, W. Climatología: con un estúdio de los climas de la tierra. México. Fondo de cultura económica, 1948. 478p.

PERAZOLLO, A. S. Cultivares de pêssegos, ameixas e nectarinas. In: ENCONTRO NACIONAL SOBRE FRUTICULTURA TEMPERADA, 2., 1999. Fraiburgo, SC, Anais..., p.70-76.

PETRI, J. L. Dormência da macieira. In: EMCAPA - Empresa Catarinense de Pesquisa Agropecuária. Manual da cultura da macieira. Florianópolis: EMPASC, 1986. Cap. 7, p.163-201.

PETRI, J. L. et al. Dormência e indução da brotação de fruteiras de clima temperado. Florianópolis: EPAGRI, 1996. 110p. (Boletim Técnico, 75). 